\title{
Disruption and Disposition in Lifelong Learning
}

\begin{abstract}
Summary
UK government policies for social inclusion through engaging with the learning society aim at repositioning people as capable participants in their social worlds. These policies at first sight appear to be aimed at a sophisticated restructuring of social contexts as well as at an enhancing of individual learning. However there is a degree of conceptual confusion within these policies. In this paper we explore some of the tensions evident in a study of a family learning centre in an English city. In the exploration we examine the extent to which the tools offered by sociocultural and activity theory (SAT) can assist in resolving that conceptual confusion and how SAT itself might need to develop in order to analyse complex and sustained forms of intervention.
\end{abstract}

\section{Social Inclusion and Lifelong Learning in the UK}

From the late 1990s the UK government has been engaged in a set of strategies aimed at enabling citizens to reposition themselves as capable participants in their communities. Under the broad banners of social inclusion and engagement in the learning society these strategies have, for example, targeted children and their families at the following stages: pre-school (Sure Start), children from five to thirteen (Children's Fund) and the fourteen to nineteen years olds (Con- nexions). These initiatives are based on strategic partnerships between existing local agencies, both statutory and voluntary. Here the key theme is neighbourhood renewal, whereby new ways of being and patterns of participation are encouraged through intervention in and with families. To support these interventions with families, existing opportunities for participation in the social sphere of local communities are being disrupted. Those opportunities which encourage responsible participation are being supported and new ones are being encouraged. These strategies appear to be a sophisticated multilevel response to the problems of social cohesion that are so much a feature of late capitalism in the West. Specifically in the UK they can be seen as investments in stabilising counterweights to the disruption of local social networks and the reduction in social capital that has been evident among the working class (Hall, 1999). However, as with any set of actions, these strategies carry with them certain historical legacies. In the UK, although the notion of 'joined-up government' is meant to enable social inclusion through inter-department collaboration, the histories of individual government departments tend to prevail in policy making. In the examples outlined so far, the prevailing 
department is education. Consequently these investments are linked directly, through the evaluation measures employed, to the UK version of the learning society which is represented by formal schooling and training.

There are some weaknesses in the relatively narrow vision of the Learning Society in operation. Not the least of these is an emphasis on learning as an individualised activity evidenced in success in knowledge reproduction on accredited courses which lead to paid employment (Ranson, Rikowski and Strain, 2001). In attempting to orchestrate major economic and social change, policy makers have focused on individuals, families and local opportunities but, it seems, they have yet to work out how the levels interact to enable the full range of identity transformations that might occur. Particularly there does not yet seem to be a worked out view of how individual learning interacts with the wider sets of distributed affordances of local social capital. It is a conceptual weakness that can perhaps only be addressed by an exploration of the complex interactions of the macro, exo, meso and microsystems in which individuals operate and the part that such interactions play in identity formation suggested by Cole (Cole, 1996).

However, in England it appears that the Neo-Liberal individualism of the image of economic man, which underpins lifelong learning, ensures a limited view of learning and learners. Here the learner is merely the consumer of opportunities in the social sphere which lead to his or her ultimate contribution to the workforce. A major element in this weakness is the parallel but competing set of discourses of learning and knowledge employed in the promotion of government policy on lifelong and family learning. The UK government in all its education policies speaks the language of individualism and marketisation and of knowledge as a commodity, yet in its social policies ac- knowledges the importance of changing patterns of participation and the need for some intervention at the level of community through support for, for example, family learning. In brief, the educational element of policy appears to be led primarily by a knowledge-driven theory of change, while those of the family and community by a social-practices version. While many of us would not see knowledge and practice as incompatible, the theory of learning driving UK educational policy is not as informed (Edwards, A., 2002) and there is a resulting conceptual conflict in lifelong learning.

Richard Edwards, for example, describes lifelong learning as 'an uncertain and troubled conceptual space' (Edwards, R., 2000: 5). It certainly does seem that a robust conceptualisation of how the layers of individual, family and community interact to allow for identity transformations which are evidenced in responsible, and if necessary critical, participation in socially cohering practices is lacking. Sociocultural and Activity Theory (SAT) (see for example Cole, 1996) arguably offers one way of conceptualising links between individuals, actions and contexts which can account for the dynamic nature of these relationships. In our study of family learning in England we have used SAT as one set of theoretical lenses through which we have tried to make sense of what is going on.

One conceptual gap in UK education policy, even when played out in the apparently less constrained arena of lifelong learning, is an understanding of learning as accomplishing (Pea, 1993). That is, learning is a matter of developing a capacity to recognise and use the resources available to promote one's actions (Clark, 1997). This analysis recognises that we inhabit a variety of activity systems (Engeström, 1999), sometimes simultaneously. For example, we may find ourselves being, at almost the same time, pro- 
fessionals, employees, friends while apparently engaged in professional activities. These different subjectivities will mean that we interpret the object of our activities in constantly shifting ways as each activity system comes into play. While these activity systems may overlap, what makes them places where we can act are the interpretations we place on the affordances for action that each system offers.

Such a view of learning places personal identity and disposition to engage with available resources at the core of lifelong learning. When considering interventions aimed at enhancing participation through, for example, engaging in opportunities for family learning, the notion of overlapping activity systems becomes crucially important. We need to understand how new patterns of participation which are developed within one setting are carried forward to other settings. It is not always entirely clear how SAT can assist us in this level of conceptualisation. However, to be true to Vygotsky's concern that theory should develop in iteration with the field, it does need to attempt to deal with this issue.

Despite Cole's analysis of how we are both shaped by and shape the worlds in which we act (Cole, 1996), understandings of how identity is manifested in intentional or deliberative action are somewhat limited within the SAT field. The problems, I shall argue, lie in both how the individual in conceptualised and how intentional action appears merely as an emergent property in systems which are able to accommodate it.

One way into SAT is to focus on individual development and see it as 'the study of the development of psychological functions through social participation in societally-organised practices' (Chaiklin, 2001: 21). As lucid a description as this is, it does not seem to take into account how those psychological functions are employed differentially in dif- ferent settings. While it allows an understanding of learning as a capacity to expand the object of one's enquiry (Engeström, 1999), it does not capture the notion of a variable disposition to engage with aspects of one's world. What hints of agency as deliberative action there are elsewhere in SAT writing, are also somewhat limited. For example, Hutchins in Daniels (2001).

\footnotetext{
"What we learn and what we know, and what our culture knows for us in the form of the structure of artefacts and social organisation are those hunks of mediating structure. Thinking consists of bringing these structures into co-ordination with each other such that they can shape (and be shaped by) each other. The thinker in this world is a very special medium that can provide co-ordination among many structured media some internal, some external, some embodied in artefacts, some in ideas, and some in social relationships." (Hutchins, 1986: 57 emphases mine)
}

\section{As Dreier so neatly summarises}

"The concrete location of individual subjects in social practice remains strangely implicit or ambiguous." (Dreier, 1999: 6)

Dreier too avoids a primary focus on individual transformation. Nonetheless his own attempt at understanding individuals' trajectories in and across these practices is helpful. Particularly so when conceptualising the kinds of learning trajectories across activity systems that one might hope to find occurring as a result of current inclusion strategies.

“...social contexts may be arranged for particular trajectories of participation in them and through them, e.g. by virtue of an internal structure of divisions and stations or an array of social contexts for the unfolding of personal life trajectories, with transitions and changing constellations of personal social practice and configurations of personal significance." (Dreier, 1999: 8) 
Dreier's contribution is important because the lack of connection between the individual and collective in activity theory is acknowledged as a difficulty (Engeström, 1999). Indeed the individual seems only a bit player and appears almost by default, perhaps when she is faced with something unexpected and is in a context which allows her to respond to it. Jay Lemke (Lemke, 2001) calls this phenomenon 'slippage', i.e. a slippage in a system which allows the individual to emerge. Slippage is a useful concept in relation to, for example, the strategies for neighbourhood renewal being employed currently in the UK, which aim to disrupt and enhance opportunities for social participation in local communities. It is also reinforced as a concept in the analyses of history in person provided by contributors to the collection put together by Holland and Lave (Holland and Lave, 2001). There it is argued that new forms of, often agentic, identities begin to take shape when existing discourses are disrupted and contentious practices emerge.

In such cases it seems that not only are new event scripts used to mediate responses to features of our worlds, but a disposition to expand the object, i.e. to see things differently is invoked. Damasio's work on disposition in neurological systems where he describes disposition as a synonym for implicit or nonconscious knowledge provides some insight into how this occurs (Damasio, 1999). Dispositions, in his analyses, are both part of our genetic endowment and our social learning and lie dormant until triggered by external events. They then help us make sense of events by shaping our interpretations of them and invoking motivations to respond in particular ways, which depend on past learning. He describes disposition as latent knowhow held at the level of the cell, which is used as we respond to changes in our environment.

\begin{abstract}
"All our memory, inherited from evolution and available at birth, or acquired through learning thereafter, in short, all our memory of things, of properties of things, of persons, of places, of events and relationships, of skills, of biological regulations, you name it, exists in dispositional form ... waiting to become an explicit image or action. Note that dispositions are not words. They are abstract records of potentialities." (Damasio, 1999: 332)
\end{abstract}

Arguably one of the challenges to a government aiming at enhancing social inclusion through disrupting and encouraging particular forms of social practices is to ensure that a disposition to engage is not concerned with contentious practices, but with what Bruner has termed cultural 'proprieties' (Bruner, 1996). This is dangerous ground for governments. It is little wonder in the UK we find that, instead of attention to generating social capital through enhancing relationships between individuals within and across the boundaries of the activity systems in which they operate, there is an emphasis on family responsibility for individual citizen making and a focus on individual performance in the workplace. Edwards is probably correct in his analysis that what is being aimed at is the production of self-reliant consumerist selves (Edwards, R., in press).

\section{Family Learning as a Case Study}

In our research on family learning centres in England we have picked up on a number of the tensions to be found in the strategic responses to social inclusion just outlined. But first a brief outline of the study and our research aims.

We are examining the impact of different forms of support for family learning on the patterns of participation and learning trajectories of those who receive the support given in the centres. The first stage of the project was a survey of family learning provision 
across England to identify key features, for example, principal source of funding and mode of operation. The survey was followed by in-depth interviews with key informants from a cross section of the survey sample. The interviews assisted the identification of the final case study sample of six family learning centres, which were selected by source of funding, mode of operation and geographical location to ensure a broad coverage of the forms of family learning support and their communities.

The case studies include a centre, now focusing on mothers and children, which was set up in the mid 1980s by the wives of coal miners involved in the strikes in the Yorkshire coal fields, an inner city drop in centre for women, a city technology centre, a local library and a centre catering for people living on a housing estate located at the outer reaches of a medium sized rural town. Using an activity theory framework we have been able to trace how the histories, professional practices and community expectations play into the ways in which identity transformations within each centre are supported. However, we are finding that we need to expand our interpretations of activity theory to deal with our analyses of individual learning trajectories beyond the centres.

We are using a range of data collection methods to gather information from workers and family members in the centres and those whose work is likely to impact on the participation of family members in other activities in the community. These methods include field notes, interviews, discussions with participants based on photographs of centre events, notes on discussions of practice at, for example, general and team meetings when we feedback our findings and analyses to workers and focus groups. These methods allow us to engage in a process of progressive focusing on the practices of the centres as activity systems and on the individual identity trajectories of those who the centres are aiming to support.

We are not seeking simple linear connections of cause and effect between experience in the centres and behaviour in other settings. Like Barbara Adam, we acknowledge how context interacts with time to render problematic simple evaluations of the impact of interventions (Adam, 1999). However, we are concerned to trace the practices of family members and their sense making as they participate in different settings. We are interested in how selves are produced and enacted in settings. These may be new environments (e.g. adult education classes) or established ones (e.g. the family). We anticipate points of conflict as individuals attempt to reposition themselves in existing systems or enter new ones and are capturing these conflicts in our research.

In exploring changing ways of being, seeing and responding we are working with a notion of identity as a construct that emerges like any other construct in the processes of approaching, interpreting and responding to the possibilities for action in a setting. It is most likely to be evident in discordance between intentional individuals and the opportunities for action available to them. We are, therefore, not suggesting that selves are merely products of discourse, but that aspects of self emerge and are confirmed in some discourses and not others. Our selves demonstrate continuity in their emergence in the practices of different settings and that occurs to the extent that patterns of participation in different settings elicit similar meanings and practices. Our selves are not simply embodied and revealed in narratives we carry with us (our self concepts) but also in our attempts at sense making, in our actions and interactions in settings i.e. in the ways in which we interpret our selves and contexts for action. It is the nature of those interactions and particularly how individual sense 
making occurs in new settings that is one of our concerns.

We will take one of the case studies to illustrate some of the policy tensions discussed earlier and at the same time identify areas where developments in SAT may usefully enhance conceptualisations of social inclusion through lifelong learning. The case is the inner city drop in centre. It has in common with all the other cases, with the exception of the city technology centre, an established history of engagement with the community it serves and a primary focus on the needs of women. The workers, who are all women, offer a range of services which include money advice, child care and short term learning projects in collaboration with, for example, adult education and community arts services. Its work within its inner city community has evolved over fifteen years. Workers in the centre come from social services, housing and money advice and volunteer backgrounds, but work fluidly and collaboratively across roles and responsibilities.

Although the centre aims at helping women to reposition themselves within their worlds so that they are able to deal with the complex demands of relationships, childcare and economic viability it does not have as a primary concern either the creation of good mothers or the production of a capable workforce. Workers refuse to intervene with children if their mothers are present and adult education opportunities are a far lower priority than short 'trips' which enable participants to see the world beyond the confines of their housing estate. Rather, the centre aims at helping participants to build up their own networks of social support though encouraging mutually supportive relationships within the centre.

“...it's the same for every individual. If you've got a friend who doesn't judge you and you know is supportive, then that can make a big difference." (centre worker)
The importance of non-judgmental support resonates throughout our interviews. Here a centre worker is describing the drop-in element of their work.

\footnotetext{
"I think that the purpose is having somewhere to go that is open, you can go in at any level really. Go in for a cup of coffee. Go in for advice. You don't need a reason to step over the door, you know in terms of using the professionals here ... you might be meeting your friend, seeing what is going on for the kids, somewhere where you can take the kids and we don't mind if they make a mess."
}

The development of this capacity to support each other is also evident in our focus group discussions and interviews with individual participants. An important theme emerging in our data seems to be that a capacity to use the resources available does not simply apply to making use of physical resources, such as how to get a loan from the money advice service. It also involves a capacity to offer support to and ask for support from others. This attribute might be termed a form of relational agency where one's capacity to engage with the world is enhanced through doing so alongside others. What the centre is doing is creating an open enough system for that kind of fluid form of relational agency to emerge. The fluidity of such relationships is important as it is clear that what is being encouraged is not dependency, but a capacity to both seek and give help when engaging with the world.

Two issues are evident here. The first is a policy matter. The centre is not engaging with the current lifelong learning agenda as played out in a concern with knowledge reproduction on accredited courses leading to employment, neither is it attempting to create self reliant consumers. Equally it is not aiming at improving the mothering skills of users. In short, although it is clearly working for social inclusion, it is operating with a 
much wider notion of what might constitute participation in societal practices. It is consequently offering a useful challenge to consumerist notions of the learning society. Particularly relevant is the way that its emphasis on a capacity for support giving is generating forms of social capital which are likely to enhance social cohesion.

The second issue raised by the centre relates to how SAT is able to conceptualise what is occurring. We've started to suggest that there are two key features enhancing the learning that is evident in the centre. The first is an emphasis on mutually supported action. The second is the openness and flexibility of the practices of the professionals who work there. In SAT terms the practitioners are creating learning zones for the users which contain within them a wide range of possibilities for action. What Valsiner terms the zone of freedom of movement (ZFM) (Valsiner, 1998) within these learning zones is relatively generous. The freedom has come in part because of the range of support offered i.e. from both the practitioners and other users, and the opportunity for the users to be both supporters and learners. In mutually supported activities the users are engaging with the dispositions of others, expanding their interpretations of events and are supported in any of the more risky aspects of responses to their interpretations. Their experiences add to the funds of knowledge held within the centre and strengthen the social capital that accrues from joint actions (Putnam, 2000).

While these processes might be described in terms of scaffolding, this is not a notion of scaffolding where it is necessarily a matter of an expert other guiding a novice towards socially acceptable forms of participation. What appears to be occurring, particularly in cases of mutual support between users, are sets of joint action on problematic objects in which both are learners. This is able to hap- pen because the relationships are established within a setting which is avowedly informal, where 'getting it wrong' is not an issue and where there is space for tentative actions, risk taking and exploration of goals. The flexibility and fluidity of roles together with an emphasis on mutual support is encouraging participation alongside others in a wide range of social practices. It would seem that Engeström's notion of the zone of proximal development as a product of new forms of societal activity (Engeström, 1987) needs to be complemented by a notion of how individuals develop the disposition to participate. Evidence from the case under discussion suggests that mutual support or relational agency is a concept worth exploring in that regard.

But what happens when the users of the centre participate in social practices in other aspects of their lives, such as the family or other community-based resources? Do these forms of societal activity similarly create learning zones in which the learning trajectories stimulated within the centre can be pursued? In our study we have envisaged the relationship between the family learning centres, families and other community resources as a set of three overlapping circles. One of our concerns has been to explore whether the overlaps between, for example, the family learning centre and other community resources offer, in Dreier's terms, an 'array of social contexts for the unfolding of personal life trajectories' (Dreier, 1999: 8).

An initial focus has been on the extent to which different services recognise what each other is trying to do so that they can offer continuity of support for users i.e. to what extent is there evidence of knotworking (Engeström, Engeström and Vähäaho, 1999). This was clearly something the centre was at pains to tackle, but it was clear that collaboration between agencies was a point of frustration for all concerned. In our feed- 
back to the centre we described a meeting attended by representatives of other local agencies in the following way.

"Researcher After the AGM (Annual General Meeting) all the users disappeared and everybody else was left. All the professionals and practitioners were left. And there was a real flurry of everyone grabbing the chance to talk to each other. It seemed like there was a desperation, but people were really glad of the opportunity to get together and make cross connections. The charge to it seemed to suggest that it was something that was actually quite important for everybody and it doesn't happen.

Centre worker No it doesn't, not usually with those people. They don't usually cross over. We have different forums, different networks like the domestic violence network, or Sure Start."

It seemed that the overlaps that did exist are largely created by the centre workers in response to crises in the lives of users. One of the practitioners described how she supported users when they interacted with other service providers.

"Sometimes it may mean that you are liasing with those other agencies as an advocate to smooth the way. You know for some people the way they present themselves is not acceptable. So actually you have to get people on (their) side ... get some understanding of where they are coming from so that they treat them better."

However it seemed that where there was a will on both sides for work in the overlaps to disrupt existing practices, there was far more potential for creating new and more open configurations which might support new identity trajectories. This observation bodes well for those strategic partnerships which take collaborative interagency work seriously. A particularly successful example of this was the relationship that was developing between the local art gallery which had a new and strong commitment to community in- volvement and the centre. The link had been set up and encouraged by a community artist who worked in both the centre and the gallery. The connection met the needs of the newly appointed curator with responsibility for community education. She described how she wanted the users of the family learning centre to feel that they were also legitimate users of the gallery.

"You come in to see the person as much as the place. By then they will have a relationship in place. Give them ownership, give them something to do ... but it will take time. I need to develop my relationship and then you will start to see a long term relationship. When they come in they will start to recognise the relationship and feel they have a voice."

Here the service provider has intended to disrupt existing patterns of participation and offer a social context for the unfolding of identity trajectories which is compatible with what is occurring within the centre. She has been enabled in doing so by the community artist who knows the users well. She is also employing a version of relational agency in the way that she describes their induction into these new practices and developing their dispositions to engage with the gallery's facilities.

Such a relational notion of disposition finds support in Damasio's work. For example, successful relationships that span and can shape opportunities for participation in more than one site of identity construction and learning are helpful in triggering dispositions to engage in new contexts. The community artist enabled participation across settings by scaffolding the users participation in new settings, recalling previous achievements and intentions and activating dispositions. 


\section{Implications for Social Inclusion Through Lifelong Learning}

What are the implications suggested by this analysis for the troubled conceptual space that is lifelong learning described by Edwards, (Edwards, R., 2000)? It does seem clear that social inclusion through participation in the learning society need not be limited to success on accredited courses. Rather learning, evidenced as a capacity to recognise and engage with available resources, occurs through a complex interweaving of individual dispositions, immediate contextual conditions and wider cultural affordances. When we unpack these links in our study, we find ourselves flagging the need for an enhanced notion of disposition to inform understandings of learning which focus on participation and particularly the take up of opportunities to participate in new forms of social practice. We are also suggesting that conceptualisations of lifelong learning should acknowledge how joint action can enrich interpretations and responses i.e. expand the object and enhance the range of possible responses to it. We are also noting that the mutuality we saw encouraged in the case study centre was enabled by the informality in roles and structures we observed there. A SAT analysis of current policy in England would support its intention to create new learning zones through disrupting and extending existing possibilities for social participation. It would however caution that such disruptions need to retain a degree of fluidity and flexibility if there is to be the space for individuals, pairs or groups to respond creatively to the opportunities provided.

However, SAT itself perhaps needs to accommodate more overtly a notion of intentional action to complement its attention to disruption in the system and expansion of the object, if it is to explicate thoroughly the relationships that obtain between individual and collective. It would also seem that the tentative steps that are occurring within the field to understand the development of new forms of participation in overlapping activity systems are well worth pursuing if the boundary crossing work of strategic partnerships is to be more than sets of responses to individual crises.

\section{References}

Adam, B. 1999. Radiated identities: in pursuit of the temporal complexity of conceptual cultural practices. In: M. Featherstone \& S. Lash (eds.): Spaces of Culture. London: Sage, 138158.

Bruner, J. 1996. The Culture of Education. Cambridge MA: Harvard University Press.

Chaiklin, S. 2001. The category of 'personality' in cultural-historical psychology. In S. Chaiklin (ed.): The Theory and Practice of Cultural-Historical Psychology. Aarhus: Aarhus University Press, 238-259.

Clark, A. 1997. Being There: putting brain, body and world together again. Cambridge MA: MIT Press.

Cole, M. 1996. Cultural Psychology. Cambridge MA: Harvard University Press.

Damasio, A. 1999. The Feeling of What Happens. New York: Harcourt Brace.

Daniels, H. 2001. Vygotsky and Pedagogy. London: Routledge.

Dreier, O. 1999. Personal Trajectories of Participation across Contexts of Social Practice. Outlines: Critical Social Studies, 1.1. 5-32.

Edwards, A. 2002. Responsible Research: ways of being a researcher. British Educational Research Journal, 28. 2. 157-168.

Edwards, R. 2000. Lifelong Learning, Lifelong Learning, Lifelong Learning: a recurrent education? In: J. Field \& M. Leicester (eds.): Lifelong Learning: education across the lifespan. London: Routledge, 3-11. 
Edwards, R. (in press) Mobilising Learning: governmentality in educational practices, Journal of Education Policy.

Engeström. Y. (1987) Learning by Expanding. Helsinki: Orienta-Konsultit.

Engeström, Y. 1999. Activity theory and individual and social transformation in Engeström, Y. et. al. (eds.) Perspectives on Activity Theory. Cambridge: Cambridge University Press, 19-38.

Engeström, Y., Engeström, R. \& Vähäaho, T. 1999. When the center does not hold: The importance of knotworking. In: S. Chaiklin, M. Hedegaard \& U. J. Jensen (eds.): Activity theory and social practice. Aarhus: Aarhus University Press, 345-374.

Hall, P. 1999. Social Capital in Britain. British Journal of Political Science, 29, 417-461.

Holland, D. \& Lave, J. 2001. (eds.) History in Person. Oxford: James Currey.

Hutchins, E. 1986. Mediation and Automatization. Quarterly Newsletter of the Laboratory of Comparative Human Cognition, April 1986. 8.2.47-58.

Lemke, J. 2001. Re: horizontal/vertical/dialogue/dialectic, XMCA discussion archive, April 2001, http://lchc.ucsd.edu/histarch/index.hmtl

Pea, R. 1993. Practices of distributed intelligence and designs for education. In: G. Salomon (ed.): Distributed Cognitions: psychological and educational considerations. Cambridge: Cambridge University Press, 47-87.

Putnam, R. 2000. Bowling Alone. New York: Touchstone.

Ranson, S., Rikowski, G. \& Strain, M. 2001. Lifelong learning for a learning democracy. In: D. Aspin et. al. (eds.): International Handbook of Lifelong Learning. Dordecht: Kluwer, 135-154.

Valsiner, J. 1998. The Guided Mind Cambridge MA: Harvard University Press. 